\title{
Neuropathologic features associated with basal forebrain atrophy in Alzheimer disease
}

Stefan J. Teipel, MD, H.-Christian Fritz, BSc, and Michel J. Grothe, PhD, for the Alzheimer's Disease Neuroimaging Initiative

Neurology ${ }^{\circledR}$ 2020;95:e1301-e1311. doi:10.1212/WNL.0000000000010192

\author{
Correspondence \\ Dr. Teipel \\ stefan.teipel@ \\ med.uni-rostock.de
}

\begin{abstract}
Objective

To study the neuropathologic correlates of cholinergic basal forebrain (BF) atrophy as determined using antemortem MRI in the Alzheimer disease (AD) spectrum.

\section{Methods}

We determined associations between BF volume from antemortem MRI brain scans and postmortem assessment of neuropathologic features, including neuritic plaques, neurofibrillary tangles (NFTs), Lewy body (LB) pathology, and TDP-43, in 64 cases of the Alzheimer's Disease Neuroimaging Initiative cohort. For comparison, we assessed neuropathologic features associated with hippocampal and parahippocampal gyrus atrophy. In addition to region of interest-based analysis, we determined the association of neuropathologic features with whole brain gray matter volume using regionally unbiased voxel-based volumetry.
\end{abstract}

\section{Results}

BF atrophy was associated with Thal amyloid phases (95\% confidence interval [CI] -0.49 to $-0.01, p=0.049)$ and presence of LB pathology ( $95 \% \mathrm{CI}-0.54$ to $-0.06, p=0.015)$, as well as with the degree of LB pathology within the nucleus basalis Meynert ( $95 \% \mathrm{CI}-0.54$ to $-0.07, p=$ 0.025). These effects were no longer significant after false discovery rate (FDR) correction. Hippocampal atrophy was significantly associated with the presence of TDP-43 pathology ( $95 \% \mathrm{CI}-0.61$ to $-0.17, p=0.003$; surviving FDR correction), in addition to dentate gyrus NFT load ( $95 \%$ CI -0.49 to $-0.01, p=0.044$; uncorrected). Voxel-based analysis confirmed spatially restricted effects of Thal phases and presence of LB pathology on BF volume.

\section{Conclusions}

These findings indicate that neuropathologic correlates of regional atrophy differ substantially between different brain regions that are typically involved in $\mathrm{AD}$-related neurodegeneration, including different susceptibilities to common comorbid pathologies. 


\section{Glossary}

$\mathbf{A D}=$ Alzheimer disease $; \mathbf{A D N I}=$ Alzheimer's Disease Neuroimaging Initiative $\mathbf{B F}=$ basal forebrain $\mathbf{C D R}=$ Clinical Dementia Rating; CERAD = Consortium to Establish a Registry for Alzheimer's Disease; CI = confidence interval; $\mathbf{C N}=$ cognitively normal; FDR = false discovery rate; $\mathbf{G M}=$ gray matter; LB = Lewy body; $\mathbf{M C I}=$ mild cognitive impairment; $\mathbf{M M S E}=$ MiniMental State Examination; MNI = Montreal Neurological Institute; MPRAGE = magnetization-prepared rapid gradient echo; MTL = medial temporal lobe; NFT = neurofibrillary tangle; NbM = nucleus basalis Meynert; ROI = region of interest; $\mathbf{S M C}=$ subjective memory concern; TIV = total intracranial volume; $\mathbf{W M}=$ white matter.

MRI-based volumetry is recognized as a topographic marker for staging and monitoring the progression of Alzheimer disease $(\mathrm{AD}) .{ }^{1}$ To understand the pathologic substrate of MRI-based volumetric changes, previous studies have investigated associations of neuropathologic measures of primary $\mathrm{AD}$ pathology and common comorbid pathologies with antemortem MRI volumetry. A systematic review identified 27 studies until mid-2015 on antemortem MRI and neuropathology. ${ }^{2}$ The large majority of these and subsequent studies focused on the hippocampus and other medial temporal lobe (MTL) structures, such as the parahippocampal gyrus and entorhinal cortex.

Besides degeneration of the hippocampus and MTL, cholinergic system degeneration is a key event in $\mathrm{AD}$ pathogenesis. ${ }^{3}$ Over the last decade, advances in MRI data analysis have enabled in vivo volumetry of the cholinergic basal forebrain $(\mathrm{BF}),{ }^{4,5}$ the central site of origin of cholinergic projections to the cerebral cortex and limbic system in the human brain. ${ }^{6}$ Studies using in vivo $\mathrm{BF}$ volumetry to study $\mathrm{BF}$ degeneration in the course of $\mathrm{AD}$ have shown that this brain region is severely atrophied in $\mathrm{AD}$ dementia and may even precede MTL degeneration in the prodromal phase of $\mathrm{AD} .{ }^{5,7}$ Neuropathologic studies suggest altered expression of neurotrophic receptors on cholinergic neurons, as well as neurofibrillary tangle (NFT) accumulation and dystrophic cell shrinkage, but not frank loss of cholinergic $\mathrm{BF}$ neurons in $\mathrm{MCI}$ and early $\mathrm{AD}$ cases. ${ }^{8}$ Whereas the main pathologic correlate of cholinergic BF degeneration in $\mathrm{AD}$ is considered to be NFT pathology, ${ }^{9}$ this brain region also shows high susceptibility to other neurodegenerative pathologies, particularly Lewy body (LB) pathology, ${ }^{10}$ but also TDP-43 pathology. ${ }^{11}$ In addition to these local pathologic changes, distinct associations between cholinergic system degeneration and accumulation of cortical amyloid pathology have been described in postmortem data, ${ }^{12}$ and these associations could recently also be demonstrated in vivo using MRI-measured BF volume and amyloid-sensitive PET imaging. ${ }^{13,14}$ However, associations between in vivo BF volume on MRI and global and local neuropathologic lesions are unexplored.

We used available antemortem MRI and postmortem neuropathologic examination data from 64 individuals enrolled in the Alzheimer's Disease Neuroimaging Initiative (ADNI) to determine neuropathologic features associated with MRI-based $\mathrm{BF}$ volume and compared these to neuropathologic correlates of more widely studied MRI-based MTL volumes. In addition, we extended the currently limited evidence on the regional specificity of neuropathology associations with whole brain gray matter (GM) volume using unbiased voxel-based volumetry.

\section{Methods}

\section{Data source}

Data used in the preparation of this article were obtained from the ADNI database (adni.loni.usc.edu/). The ADNI was launched in 2003 by the National Institute on Aging, the National Institute of Biomedical Imaging and Bioengineering, the Food and Drug Administration, private pharmaceutical companies, and nonprofit organizations, with the primary goal of testing whether neuroimaging, neuropsychologic, and other biologic measurements can be used as reliable in vivo markers of $\mathrm{AD}$ pathogenesis. A fuller description of $\mathrm{ADNI}$ and up-todate information is available at adni-info.org.

\section{Standard protocol approvals, registrations, and patient consents}

All procedures performed in the ADNI studies involving human participants were in accordance with the ethical standards of the institutional research committees and with the 1964 Helsinki declaration and its later amendments. Written informed consent was obtained from all participants and/or authorized representatives and the study partners before any protocol-specific procedures were carried out in the ADNI studies.

\section{Study participants}

We retrieved the last available MRI scans of 64 ADNI participants who had come to autopsy between 2007 and 2017. Detailed inclusion criteria for the antemortem diagnostic categories can be found at the ADNI web site (adni.loni.usc.edu/ methods/). Briefly, cognitively normal (CN) participants had Mini-Mental State Examination (MMSE) scores between 24 and 30 (inclusive); had a Clinical Dementia Rating (CDR) score of 0 ; did not have depression, mild cognitive impairment (MCI), or dementia; and reported no subjective memory concerns (SMCs). One participant fulfilled identical criteria to the $\mathrm{CN}$ cases with the exception that he had a self-reported persistent memory complaint quantified by the Cognitive Change Index (with a total score from the first 12 items $\geq 16$ ). As this participant lacked objective cognitive decline, he was integrated into the $\mathrm{CN}$ group. Participants with MCI had 
MMSE scores between 24 and 30 (inclusive); a SMC reported by the participant, informant, or clinician; objective memory loss measured by education-adjusted scores on delayed recall; $\mathrm{CDR}=0.5$; absence of significant levels of impairment in other cognitive domains; essentially preserved activities of daily living; and an absence of dementia. All MCI cases were classified as amnestic MCI according to the ADNI guidelines. At inclusion into the $\mathrm{ADNI}$ cohort, patients with $\mathrm{AD}$ dementia had initial MMSE scores between 20 and 26 (inclusive), had a CDR $=0.5$ or 1.0 with impaired activities of daily living, and fulfilled National Institute of Neurological and Communicative Disorders and Stroke-Alzheimer's Disease and Related Disorders Association criteria for clinically probable AD. ${ }^{15}$

\section{Neuropathological assessments}

All neuropathologic evaluations in the ADNI cohort are performed through the central laboratory of the ADNI neuropathology core (adni.loni.usc.edu/about/\#core-container) and assess a wide range of $\mathrm{AD}$ and non- $\mathrm{AD}$ neuropathologic lesions, including both established neuropathologic criteria as well as detailed regional assessments within 22 cortical and subcortical brain regions. ${ }^{16}$ The neuropathologic procedures follow previously established guidelines ${ }^{17}$ that are captured in the format of the Neuropathology Data Form Version 10 of the National Alzheimer Coordinating Center (alz.washington.edu/ NONMEMBER/NP/npform10.pdf).

We used established rating scales for $\mathrm{AD}$ neuropathologic change represented by Thal amyloid phases, Braak NFT staging, and the Consortium to Establish a Registry for Alzheimer's Disease (CERAD) score for density of neuritic plaques, and further assessed whether comorbid LB pathology, TDP-43 pathology, or hippocampal sclerosis was present or not. In addition, we used regional neuropathologic rating scores for the nucleus basalis Meynert ( $\mathrm{NbM})$, dentate gyrus, entorhinal cortex, and parahippocampal gyrus, corresponding to our MRIbased regions of interest (ROIs) in the BF and the MTL. The ADNI autopsy data lack an assessment of CA1 neuron loss so that we did not include CA1 in our evaluation. $\mathrm{NbM}$ pathology was sampled on a coronal slice at the level of the crossing of the anterior commissure and was not selective for cholinergic neuron populations within this area. Therefore, we did not include $\mathrm{NbM}$ neuronal loss scores into our analysis. The dentate gyrus and parahippocampal gyrus were both sampled on a coronal section at the level of the lateral geniculate nucleus. The entorhinal cortex was sampled on a separate, more anterior section. Within the target regions, we focused on scores for neuronal loss, NFT, neuritic plaques, TDP-43 immunoreactive neuronal cytoplasmic inclusions, and LB pathology, assessed on semiquantitative scales (none, mild, moderate, and severe). Further details on the neuropathologic assessments can be found in the supplementary materials section (supplementary data available from Dryad, doi.org/10.5061/dryad.dfn2z34x6).

\section{Neuropsychological assessment}

The MMSE ${ }^{18}$ was available to assess degree of cognitive impairment, and established ADNI composite scores for episodic memory and executive function were used for the evaluation of domain-specific cognitive performance. ${ }^{19,20}$

\section{Imaging data acquisition}

MRI data were acquired on multiple $1.5 \mathrm{~T}$ and $3 \mathrm{~T}$ MRI scanners using scanner-specific T1-weighted sagittal 3D magnetization-prepared rapid gradient echo (MPRAGE) sequences. In order to increase signal uniformity across the multicenter scanner platforms, original MPRAGE acquisitions in $\mathrm{ADNI}$ undergo standardized image preprocessing correction steps. Detailed information on the different imaging protocols employed across ADNI sites and standardized image preprocessing steps can be found on the ADNI website (adni.loni.usc.edu/methods/).

\section{Imaging data processing}

Imaging data were processed by using statistical parametric mapping (SPM12, Wellcome Trust Center for Neuroimaging) and the CAT12.3 toolbox (dbm.neuro.uni-jena.de/ cat) implemented in MATLAB R2018a (MathWorks, Natick, MA). First, MRI scans were automatically segmented into GM, white matter (WM), and CSF partitions of $1.5 \mathrm{~mm}$ isotropic voxel size using the Adaptive Maximum A Posterior (AMAP) segmentation routine of the CAT12 toolbox. The resulting GM and WM partitions of each participant in native space were then high-dimensionally registered to the Montreal Neurological Institute (MNI) reference template using the DARTEL algorithm. ${ }^{21}$ Individual flow fields resulting from the DARTEL registration to the reference template were used to warp the GM segments and voxel values were modulated for volumetric changes introduced by the highdimensional normalization, such that the total amount of GM volume present before warping was preserved.

\section{Extraction of imaging features from BF and hippocampal ROIs}

The cholinergic nuclei are not directly visible on current structural MRI contrasts and no comprehensive set of external landmarks has been identified that could be used for indirect manual delineation of the cholinergic BF on MRI scans. In the current study, we localized the cholinergic space of the BF based on a cytoarchitectonic map of BF cholinergic nuclei in MNI space, derived from combined histology and MRI of a postmortem brain, as described previously. ${ }^{4}$

An ROI mask for the hippocampus was obtained by manual delineation of the hippocampus in the reference template following the harmonized protocol for hippocampus segmentation. ${ }^{22}$

The parahippocampal gyrus ROI was derived from the corresponding label in the "Hammers" maximum probability structural atlas, ${ }^{23}$ which includes both the parahippocampal gyrus proper and the entorhinal cortex.

Individual GM volumes of the ROIs were extracted automatically from the warped GM segments by summing up the 
Table Sample characteristics at time of last MRI

\begin{tabular}{llllll}
\hline $\begin{array}{l}\text { Clinical diagnosis } \\
\text { at last MRI }\end{array}$ & F/M $^{\mathbf{a}}$ & $\begin{array}{l}\text { Age, y, mean } \\
(\text { SD), range }\end{array}$ & $\begin{array}{l}\text { MMSE, } \\
\text { mean (SD) }\end{array}$ & $\begin{array}{l}\text { Education, } \mathbf{y}, \\
\text { mean (SD) }\end{array}$ & $\begin{array}{l}\text { Time between MRI and death, } \\
\mathbf{y}^{\mathbf{b}} \text { average (SD), range }\end{array}$ \\
\hline Controls & $3 / 2$ & $80.8(7.7), 68-88$ & $28.4(3.1)$ & $18.0(3.46)$ & $2.1(2.4), 0.5-4.1$ \\
\hline $\mathrm{MCl}$ & $1 / 10$ & $84.4(4.4), 75-90$ & $23.6(4.0)$ & $14.82(2.3)$ & $2.6(1.4), 0.5-7.1$ \\
\hline AD dementia & $10 / 36$ & $80.0(6.8), 58-92$ & $17.1(6.1)$ & $16.2(2.4)$ & $2.0(1.8), 0.1-7.8$ \\
\hline
\end{tabular}

Abbreviations: $\mathrm{AD}=$ Alzheimer disease; $\mathrm{MCl}=$ mild cognitive impairment; $\mathrm{MMSE}=$ Mini-Mental State Examination .

${ }^{a}$ Not significantly different between groups, $\chi^{2}(2 d f)=5.2, p=0.08$.

${ }^{\mathrm{b}}$ Not significantly different between groups, $F_{2,59}=2.0, p=0.15$.

c Significantly different between groups, $F_{2,59}=13.2, p<0.001$.

${ }^{\mathrm{d}}$ Not significantly different between groups, $F_{2,59}=2.9, p=0.064$.

e Not significantly different between groups, $F_{2,59}=0.41, p=0.67$.

modulated GM voxel values within the respective ROI masks in the reference space. For further analyses, the extracted regional GM volumes were scaled by the total intracranial volume (TIV), calculated as the sum of total volumes of the GM, WM, and CSF partitions.

\section{Statistics}

All variables used for analysis, except the volume measures, were directly retrieved from the ADNI database. Statistical analyses were conducted with $\mathrm{R}$ (The $\mathrm{R}$ Foundation for Statistical Computing) as implemented in R Studio, Version 1.1.453, unless otherwise specified.

Volume measurements were compared between clinical diagnostic groups using analysis of covariance models, controlling for age at MRI, sex, scanner field strength, and the temporal interval between MRI and death (considering a $p$ value of $<0.05$ as significant).

Associations between neuropathologic scores and volume measures were analyzed using partial correlations, controlling for age, sex, MRI scanner field strength, and the temporal interval between MRI and death. In addition, associations between neuropathologic scores and neuropsychological measures were analyzed using partial correlation, controlling for age, sex, and the temporal interval between the last cognitive testing and death. We report results at a $p$ value $<0.05$, uncorrected for multiple comparisons, as well as following false discovery rate (FDR) correction. $^{24}$

Figure 1 Partial correlations between regional volumes and global neuropathologic scores

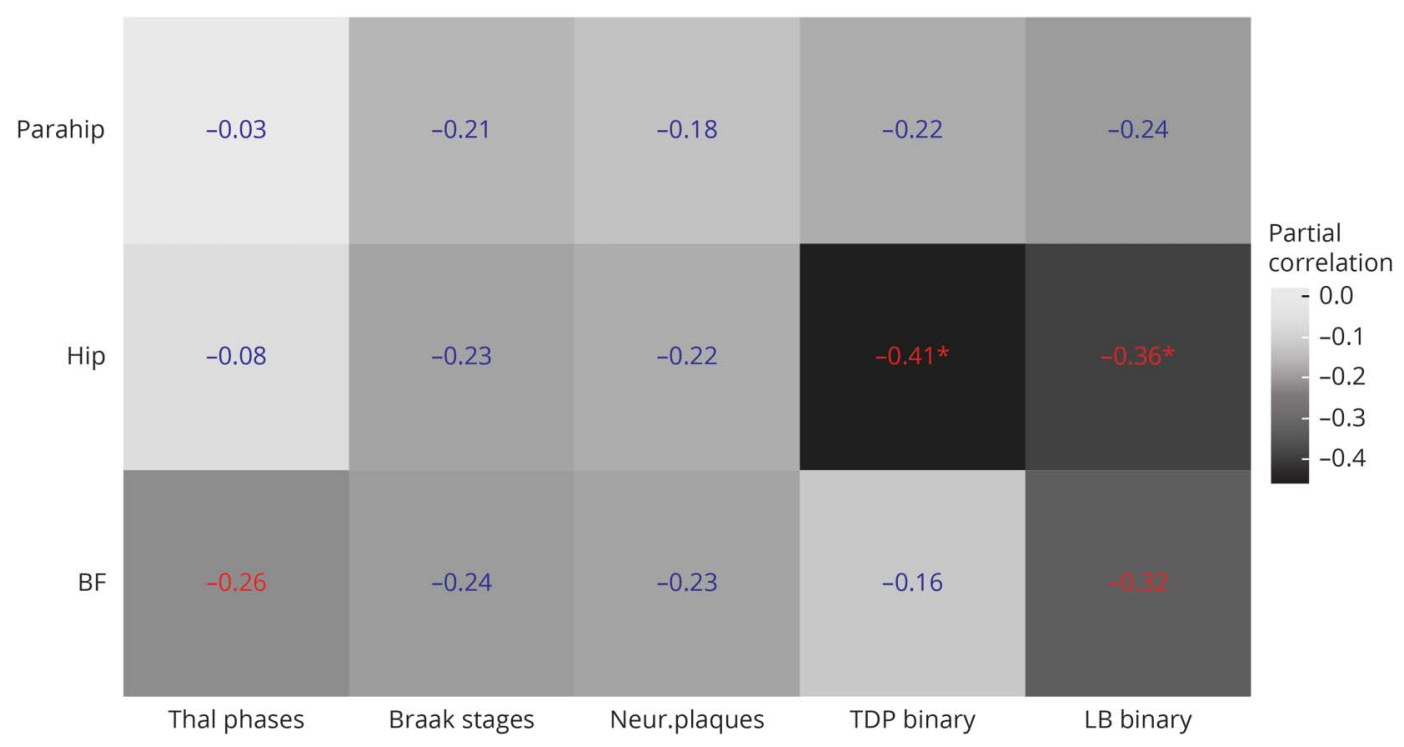

Partial correlations between regional volumes and global neuropathologic scores, controlling for age, sex, and interval between MRI and death. Numbers in blue indicate nonsignificant effects, numbers in red indicate significant effects at a $p$ value $<0.05$, uncorrected for multiple comparisons, and numbers in red with an * indicate significant effects at a false discovery rate-corrected $p$ value $<0.05$. All volumes are normalized to the individual total intracranial volume. BF = basal forebrain volume according to ref. 4; Braak stages = Braak stages for neurofibrillary pathology; Hip = hippocampal volume according to ref. 22; LB binary = presence of Lewy body pathology (assessed according to dementia with Lewy bodies criteria as brainstem predominant, limbic, neocortical, amygdala predominant), yes/no; Neur. plaques = Consortium to Establish a Registry for Alzheimer's Disease score for density of neocortical neuritic plaques; Parahip = parahippocampal gyrus from the Hammers atlas ${ }^{23}$; TDP binary = presence of TDP-43 pathology (assessed in the following regions: spinal cord, amygdala, hippocampus, entorhinal cortex, neocortical), yes/no; Thal phases = Thal phases for amyloid plaques. 


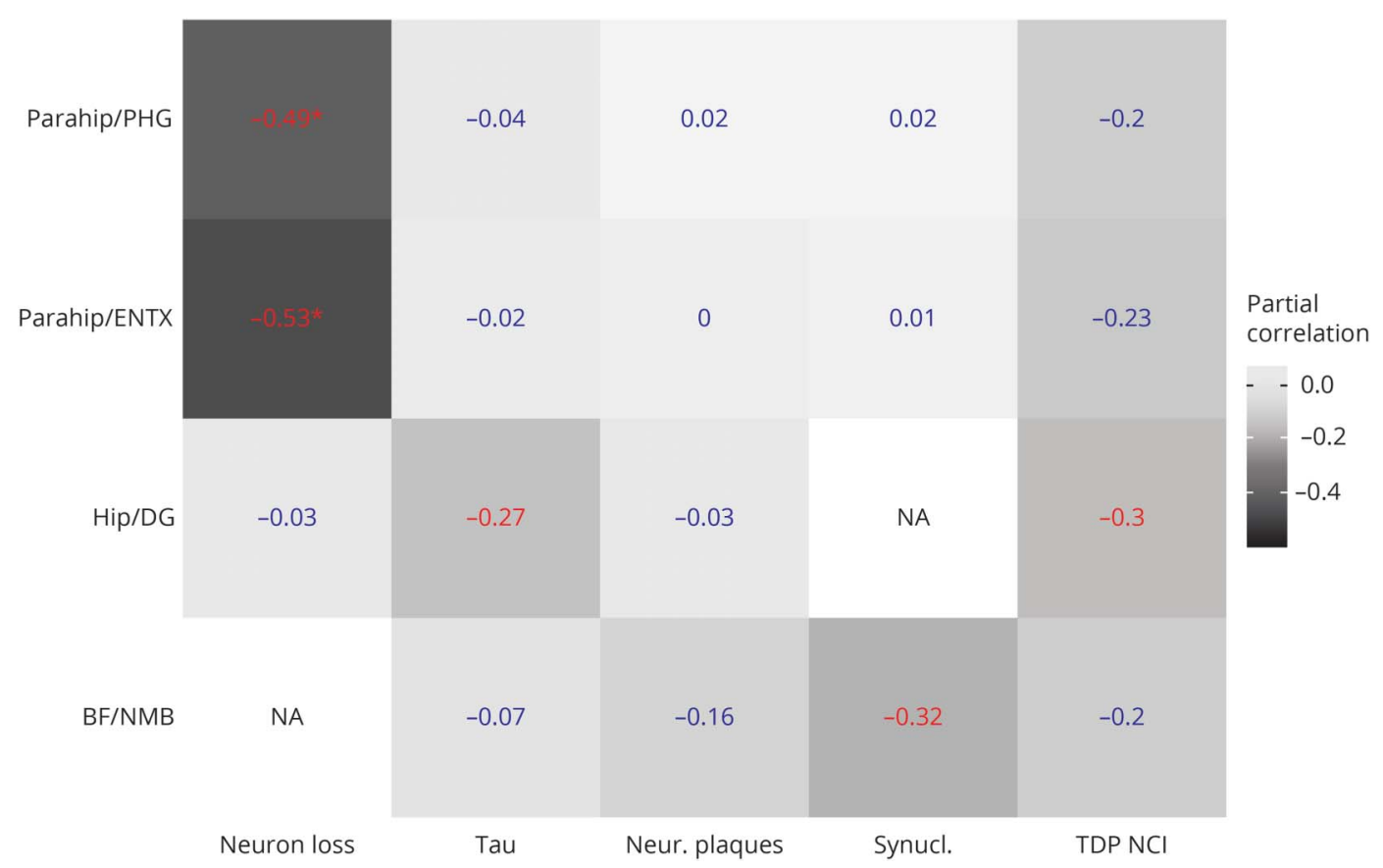

Partial correlations between regional volumes and neuropathologic scores within the brain ROIs, controlling for age, sex, scanner field strength, and temporal interval between MRI and death. Numbers in blue indicate nonsignificant effects, numbers in red indicate significant effects at a $p$ value $<0.05$, uncorrected for multiple comparisons, and numbers in red with an * indicate significant effects at a false discovery rate-corrected $p$ value $<0.05$. All volumes are normalized to the individual total intracranial volume. $\mathrm{BF}=$ basal forebrain volume according to ref. $4 ;$ Hip = hippocampal volume according to ref. $22 ; \mathrm{DG}=$ dentate gyrus (autopsy region); ENTX = entorhinal cortex (autopsy region); NA = not assessed; NMB = nucleus basalis of Meynert (autopsy region); Neur. Plaques = neuritic plaques ( 0 [none] to 3 [ $\geq 20$ per visual field]); Neuron loss = neuronal loss ( 0 [none] to 3 [severe]); Parahip = parahippocampal gyrus volume from the Hammers atlas 3 ; PHG = parahippocampal gyrus (autopsy region); Synucl. = Lewy bodies (a-synuclein-positive inclusions) $(0$ [none] to $3[>10$ or numerous LB inclusions per $\times 10$ field]); Tau $=$ neurofibrillary tangles $(0$ [none] to 3 [ $\geq 20$ per visual field]); TDP NCI $=$ TDP-43 immunoreactive neuronal cytoplasmatic inclusions ( 0 [none] to 3 [ $>10$ or numerous TDP-43 inclusions per $\times 10$ field]).

In a complementary voxel-wise analysis in SPM12, we determined the effect of neuropathologic scores that showed significant associations with ROI volumes on whole brain GM changes. The analyses were controlled for age at MRI, sex, MRI scanner field strength, and the temporal interval between MRI and death, and results are reported using a voxel-wise threshold of $p<0.001$, uncorrected.

\section{Data availability}

Neuropathologic data, demographic data, and MRI scans are available through the ADNI data repository, accessible free of cost after registration (adni.loni.usc.edu/data-samples/access-data). Volumetric measures of $\mathrm{BF}$ and hippocampus have been obtained from the MRI scans using established processing pipelines as described above. The anonymized volumetric measures containing ADNI cohort identifiers will be shared by request from any qualified investigator.

\section{Results}

\section{Sample characteristics}

Two participants, one with a clinical diagnosis of $\mathrm{AD}$ dementia and one with a clinical diagnosis of MCI, were excluded from further analysis because the processing of their MRI scans yielded inaccurate segmentation, most likely due to the severe degree of atrophy. The remaining 62 participants had received the following clinical diagnoses at the time of their last MRI scan: 5 CN older participants (1 person reported SMCs), 11 participants with $\mathrm{MCI}$, and 46 participants with $\mathrm{AD}$ dementia. Diagnostic groups did not differ significantly with respect to age, sex, education, or the temporal interval between MRI scan and death (on average 2.1 [SD 1.8] years; range 0.1-7.8 years). As expected, MMSE scores differed significantly between groups. The demographic details can be found in the table. Clinical diagnosis was significantly associated with $\mathrm{BF}$ and hippocampal $\left(F_{2,59}>3.1, p<0.05\right)$ but not parahippocampal gyrus volumes. The significant effects were no longer preserved after controlling for age, sex, and MRI scanner field strength.

\section{Neuropathologic characteristics}

With respect to neuropathologic scores in the selected brain regions, the $\mathrm{NbM}$ showed a high degree of NFT with a relatively low degree of neuritic plaques. Similarly, the parahippocampal gyrus had a high degree of NFT, but a low degree of neuronal loss and a moderate degree of neuritic plaques. The entorhinal cortex had a high degree of NFT, and a moderate to high degree of neuritic plaques and neuronal loss, whereas the dentate gyrus had a comparably low degree of NFT, neuritic plaques, and neuronal loss. Local $\alpha$-synuclein and TDP-43 was 
Figure 3 Presence of TDP-43 pathology and gray matter volume
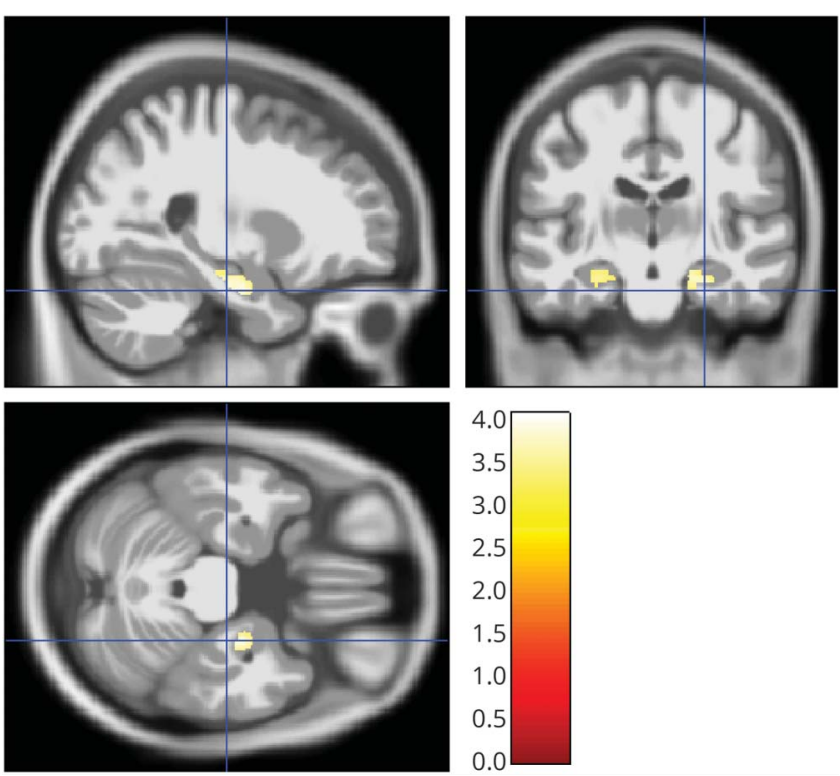

Sagittal, coronal, and axial view (right of image is right of brain) of clusters of at least 20 voxels surpassing a significance threshold of 0.001 , uncorrected for multiple comparisons, for the effect of TDP-43 pathology (absent vs present) on gray matter volume, controlling for age, sex, total intracrania volume, and the temporal interval between MRI scan and death. The color bar in the right lower corner indicates the T values.

detected only in a subgroup of cases, with TDP-43 being absent in the $\mathrm{NbM}$ (with the exception of a single case) and $\alpha$-synuclein being absent in the dentate gyrus.

\section{Associations between global neuropathologic scores and BF and MTL volumes}

Detailed nuisance-controlled partial correlations between global neuropathologic scores and MRI volumes can be found in figure 1. Thal amyloid phases and presence of LB pathology were significantly associated with $\mathrm{BF}$ volume $(r=-0.26[95 \%$ confidence interval (CI) -0.49 to -0.01$]$ and $r=-0.32[95 \%$ CI -0.54 to -0.06$]$, respectively), but effects were not significant after FDR correction. Braak tangle stages and CERAD score for neuritic plaques were not significantly associated with BF or MTL volumes. Presence of TDP-43 and LB pathology were significantly associated with hippocampal volume $(r=$ $-0.41[-0.61$ to -0.17$]$ and $r=-0.36[-0.57$ to -0.11$])$, both surviving FDR correction at $p<0.05$. Hippocampal sclerosis was only present in 5 cases (unilateral in 3 cases, laterality not assessed in 2 cases). The association between hippocampal volume and presence of TDP-43 pathology remained significant even after additionally controlling for the presence of hippocampal sclerosis $(r=-0.35[-0.56$ to -0.10$])$.

\section{Associations between regional neuropathologic scores and BF and MTL volumes}

Detailed associations can be found in figure 2. BF volume was significantly associated with the degree of LB pathology in the
$\mathrm{NbM}(r=-0.32$ [95\% CI -0.54 to -0.07$])$ and hippocampal volume was significantly correlated with dentate gyrus NFT ( $r=$ -0.27 [95\% CI -0.49 to -0.01$])$ and TDP-43 scores $(r=-0.30$ $[95 \% \mathrm{CI}-0.52$ to -0.04$])$. These effects were no longer significant after FDR correction. The parahippocampal gyrus volume (including the entorhinal cortex) was significantly associated with neuronal loss ratings in the entorhinal cortex $(r=-0.53$ [95\% CI -0.69 to -0.31$])$ and parahippocampal gyrus $(r=0.49[95 \% \mathrm{CI}$ 0.67 to -0.26$]$ ), surviving FDR correction at $p<0.05$.

\section{Neuropathologic scores and whole brain GM analysis}

In voxel-based regression analyses across the whole brain, controlling for age, sex, TIV, and interval between MRI and death, presence of LB pathology was associated with reduced GM volume in anterior cingulate GM and lateral temporal and insular cortex at $p<0.001$. Presence of TDP-43 pathology (vs no TDP-43 pathology) was associated with reduced GM volume in left and right hippocampus (figure 3 ). We found no significant voxel-wise effect of Thal phases on GM volumes at $p<0.001$. However, in a spatially restricted analysis within the BF mask only and applying a more liberal threshold of $p<$ 0.01 , we found significant effects of both Thal phases and presence of LB pathology, but not of TDP-43, indicating consistent effects with the ROI analyses for this region (figure 4, A and B).

In a complementary analysis, ${ }^{25}$ we did not find significant effects of Braak stages on GM volume at $p<0.001$. Dentate gyrus NFT score showed no significant association with GM volumes at $p<0.001$. Restricting the search region to the bilateral hippocampus and applying a more liberal threshold of $p<0.01$, we found significant effects of dentate gyrus NFT score on bilateral posterior hippocampal volume, most pronounced in the right hippocampus (figure 4C). Entorhinal cortex neuron loss showed a significant association with reduced GM in extended MTL areas (figure 5), and very similar regional associations were observed for parahippocampal gyrus neuron loss (data not shown). Adding field strength of MRI scanner as additional covariate did not markedly alter the results.

\section{Association of neuropathologic scores and neuropsychological measures}

We found a significant association of the degree of LB pathology in the $\mathrm{NbM}$ with $\mathrm{ADNI}$ executive function score $(r=$ -0.29 [95\% CI -0.52 to -0.01$]$ ), but no effect on ADNI memory or MMSE score. NbM NFT scores were not correlated with any of the 3 cognitive measures. Thal amyloid phases, Braak stages, CERAD neuritic plaque scores, and presence of LB pathology were significantly associated with global cognition, memory, and executive function. Except for the association between CERAD neuritic plaque score and executive function, these effects were preserved after FDR correction. In contrast, presence of TDP-43 pathology was not significantly associated with any of the cognitive outcomes. Detailed results are shown in figure 6. 


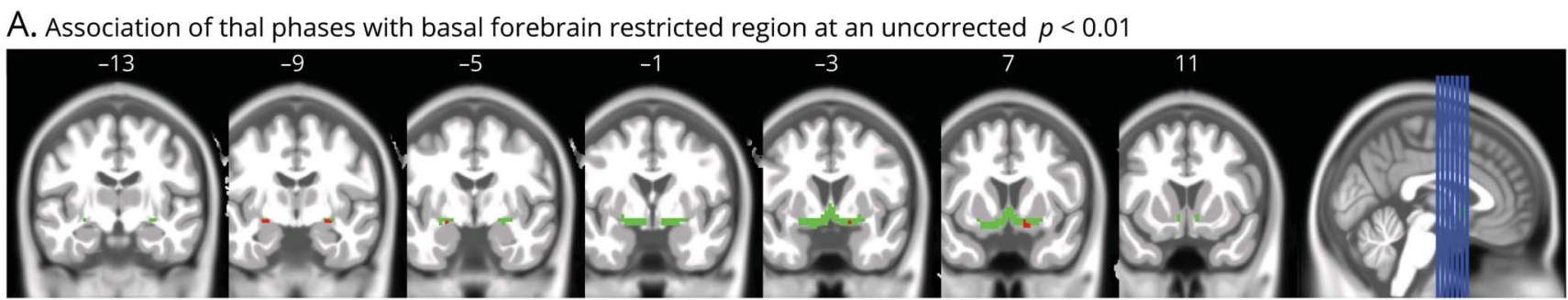

B. Association of Lewy body pathology with basal forebrain restricted region at an uncorrected $p<0.01$

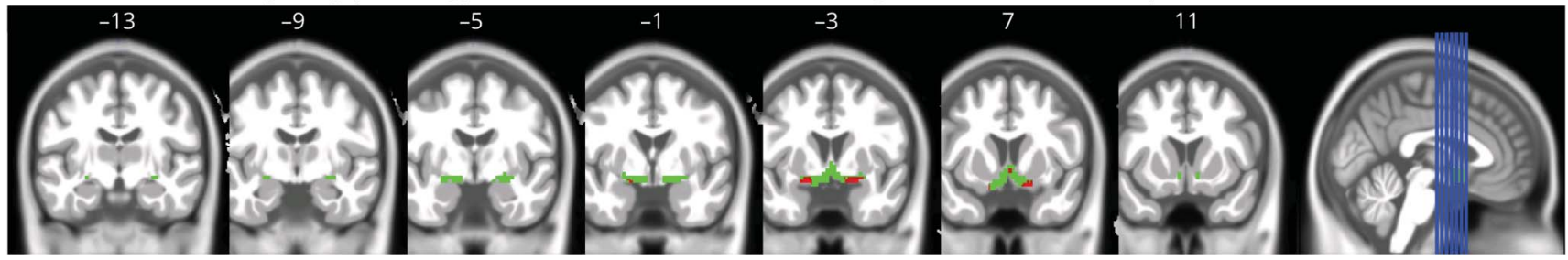

C. Association of dentate gyrus NFT score with bilateral hippocampus restricted region at an uncorrected $p<0.01$

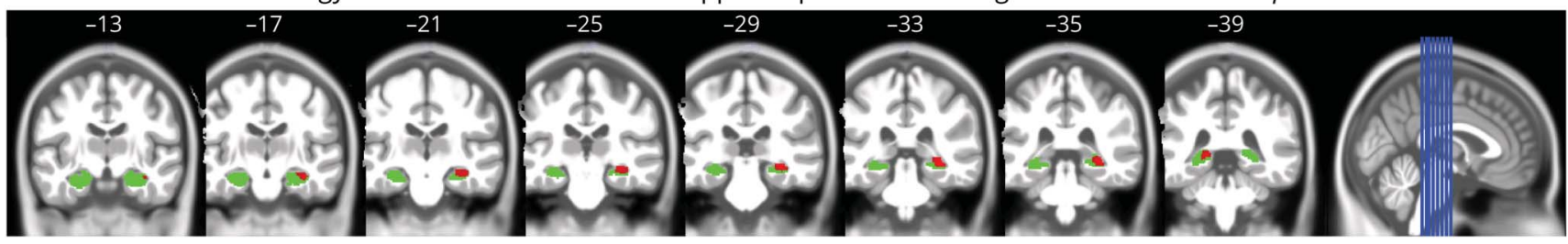

(A) Association of Thal phases with basal forebrain restricted region at an uncorrected $p<0.01$. Coronal view (right of image is right of brain) of clusters of at least 5 voxels (red) surpassing a significance threshold of 0.01 , uncorrected for multiple comparisons, for the effect of Thal phases within the basal forebrain search region (in green), controlling for age, sex, total intracranial volume (TIV), and the temporal interval between MRI scan and death. Numbers on the top indicate the y-coordinate in Montreal Neurological Institute (MNI) space (anterior posterior direction). (B) Association of Lewy body pathology with basal forebrain restricted region at an uncorrected $p<0.01$. Coronal view (right of image is right of brain) of clusters of at least 5 voxels (red) surpassing a significance threshold of 0.01 , uncorrected for multiple comparisons, for the effect of Lewy body pathology (absent vs present) within the basal forebrain search region (in green), controlling for age, sex, TIV, and the temporal interval between MRI scan and death. Numbers on the top indicate the $y$-coordinate in MNI space (anterior posterior direction). (C) Association of dentate gyrus neurofibrillary tangle (NFT) score with bilateral hippocampus restricted region at an uncorrected $p<0.01$. Coronal view (right of image is right of brain) of clusters of at least 10 voxels (red) surpassing a significance threshold of 0.01 , uncorrected for multiple comparisons, for the effect of dentate gyrus NFT score within the hippocampus search region (in green), controlling for age, sex, TIV, and the temporal interval between MRI scan and death. Numbers on the top indicate the y-coordinate in MNI space (anterior posterior direction).

\section{Discussion}

At an uncorrected level of significance, Thal amyloid phases were associated with $\mathrm{BF}$ volume. At the same time, the $\mathrm{NbM}$ itself exhibited very little neuritic plaques. Thus the degree of atrophy of the $\mathrm{NbM}$ was associated with amyloid pathology in cortical areas, but not with plaque load in the NbM itself. This agrees with postmortem studies that showed distinct associations between cholinergic system degeneration and accumulation of cortical amyloid pathology. ${ }^{12}$ Similarly, in vivo studies could also demonstrate a regionally focused association of cortical amyloid accumulation with BF volume using amyloid-sensitive PET imaging and MRI-measured BF volume. $^{13,14}$ Together, these findings suggest that $\mathrm{BF}$ atrophy on MRI associates with remote cortical amyloid pathology rather than amyloid load within the BF itself, which could reflect neurotoxic effects of cortical amyloid pathology mediated by uptake of $\beta$-amyloid proteins into cholinergic nerve terminals or a disruption of nerve growth factor signaling. ${ }^{8,26}$ In another chain of events, findings from experimental animal studies suggest that cholinergic degeneration may contribute to increased accumulation of cortical amyloid pathology due to altered amyloid processing and clearance mechanisms subserved by cholinergic signaling. ${ }^{27}$ Future in vivo studies assessing the respective biomarkers longitudinally may help to gain more insight into the directionality of the interactions between cholinergic BF atrophy and cortical amyloid pathology in the human brain.

$\mathrm{BF}$ volume was also related to the general presence of $\mathrm{LB}$ pathology as well as to the degree of LB pathology in the $\mathrm{NbM}$. One reason for the strong effect of LB pathology may be its high prevalence in the current cohort, where $31 \%$ of cases exhibited neocortical and 19\% limbic predominant LB pathology, consistent with the reportedly high comorbidity of $\mathrm{AD}$ and $\mathrm{LB}$ pathology. ${ }^{28}$ In a combined analysis, the effect of $\mathrm{NbM}$ LB pathology on BF volume rendered the effect of Thal phases nonsignificant (supplementary data available from Dryad, doi.org/10.5061/dryad.dfn2z34x6), suggesting a stronger association of LB pathology than cortical amyloid pathology with $\mathrm{BF}$ volume decline.

Of note, none of the associations between neuropathologic lesions and BF volume survived FDR correction. This 
Figure 5 Entorhinal cortex neuron loss and gray matter volume
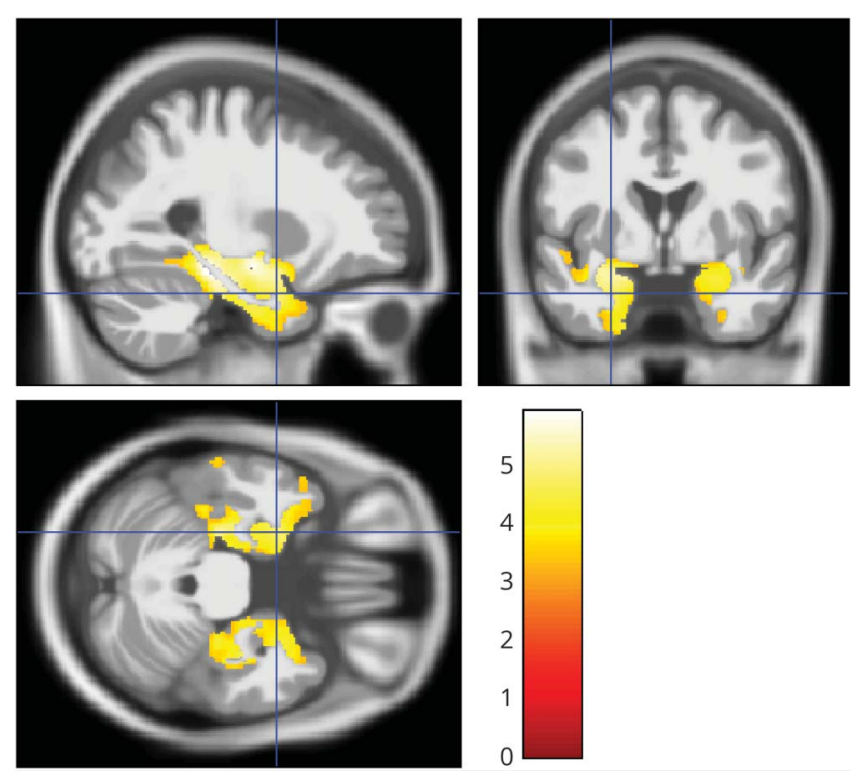

Sagittal, coronal, and axial view (right of image is right of brain) of clusters of at least 20 voxels surpassing a significance threshold of 0.001 , uncorrected for multiple comparisons, for the effect of entorhinal cortex neuron loss on gray matter volume, controlling for age, sex, total intracranial volume, and the temporal interval between MRI scan and death. The color bar in the right lower corner indicates the $T$ values.

indicates only moderate effect sizes and some risk of falsepositive findings. Therefore, in the absence of previous studies on neuropathologic correlates of BF atrophy, these findings require independent confirmation.

BF volume was not associated with NFT load in the NbM. NFTs have been found to occur already at Braak stage I in the BF cholinergic neurons, ${ }^{29}$ and NFT pathology in the NbM was found already in persons without dementia in their third decade of life, followed by increasing morphologic alterations of axons and cellular tangles preceding neuronal loss. ${ }^{30}$ In agreement with the early build-up of NFT pathology in the $\mathrm{NbM}$ in previous studies, $70 \%$ of our cases had semiquantitative ratings indicating moderate to severe NFT pathology in the NbM. Thus, the limited variation in NFT levels may have obscured possible associations between NbM NFT load and BF volume. BF neuron loss is consistently being found in advanced stages of $\mathrm{AD}$, but evidence points to dystrophic shrinkage and dysfunction of cholinergic neurons rather than to a significant reduction of cholinergic neuron numbers in the $\mathrm{BF}$ in prodromal and early $\mathrm{AD}$ stages. ${ }^{31}$ In the current study, we could not specifically analyze associations between $\mathrm{BF}$ volume and cholinergic cell loss as the available neuropathologic sampling of the $\mathrm{NbM}$ did not differentiate between cholinergic and noncholinergic neurons.

Presence of LB pathology was associated with worse cognitive performance, and the degree of LB pathology in the NbM was specifically associated with executive dysfunction. This agrees with the role of cholinergic BF integrity for executive function found in clinical studies.

In contrast to the $\mathrm{BF}$, hippocampal and parahippocampal gyrus atrophy was not associated with Thal amyloid phases. Thal phases include both diffuse and dense neuritic plaques, and previous studies found no association of diffuse plaques with total brain ${ }^{32}$ or hippocampal ${ }^{33}$ volumes. Thal phases were also not found to correlate with antemortem cognition. ${ }^{34}$ However, even CERAD score for density of neuritic plaques was not associated with hippocampal or parahippocampal gyrus volumes in our study, different from previous studies that reported associations of neuritic plaques with whole brain and MTL volumes. ${ }^{2}$ Our current findings, however, agree with findings from in vivo neuroimaging studies reporting a lack of association between PET-measured cortical amyloid load and hippocampal atrophy. ${ }^{13,14}$

Consistent with one previous study using antemortem $\mathrm{MRI}^{35}$ and several studies using postmortem MRI, ${ }^{36,37}$ we found a significant association between neuronal loss ratings in MTL regions and MTL volumes, both using ROI and voxel-based analyses. In addition, NFT score in the dentate gyrus was significantly associated with hippocampal volume. Significant associations of NFTs with hippocampal volume agree with previous studies. ${ }^{25,38}$ However, both presence of TDP-43 pathology in any location and the degree of TDP-43 pathology in the dentate gyrus were associated with MTL volumes as well, in agreement with earlier reports. ${ }^{39}$ Interestingly, the association with TDP-43 pathology even remained when controlling for hippocampal sclerosis and Braak tau stages, and additional voxel-based analysis revealed that the effect of TDP-43 was regionally specific for the bilateral hippocampus. The effect of TDP-43 was only reduced when accounting for local NFT load in the dentate gyrus. This finding agrees with a central role of comorbid TDP-43 pathology for hippocampal atrophy within the $\mathrm{AD}$ spectrum. ${ }^{40,41}$ In contrast, we did not find an association of TDP-43 pathology with BF atrophy in our study. In fact, there was only a single patient in our cohort who exhibited TDP-43 pathology in the NbM, and interestingly this patient also had hippocampal sclerosis. TDP-43 pathology in the BF has recently been reported to accompany hippocampal sclerosis, but not $\mathrm{AD}$ or other neurodegenerative diseases. ${ }^{11}$ In the current cohort, TDP-43 pathology was present at least to a mild extent in $37 \%$ of cases, which agrees with the prevalence of TDP-43 in previous studies, ranging between $33 \%$ and $52 \%$ in cases with $\mathrm{AD}$ pathology. ${ }^{40,42-44}$ Also consistent with these previous studies, the regional distribution of TDP-43 inclusions in the current sample was almost exclusively focused on MTL regions, including hippocampus, entorhinal cortex, and amygdala, and only 6 patients had neocortical TDP-43 inclusions in addition to MTL involvement. These data underscore the strong association of regional distribution of TDP-43 inclusions with the regional pattern of brain atrophy, consistent with previous reports on amygdala and hippocampal involvement with TDP-43 pathology ${ }^{45}$ and the current discussion on limbic 


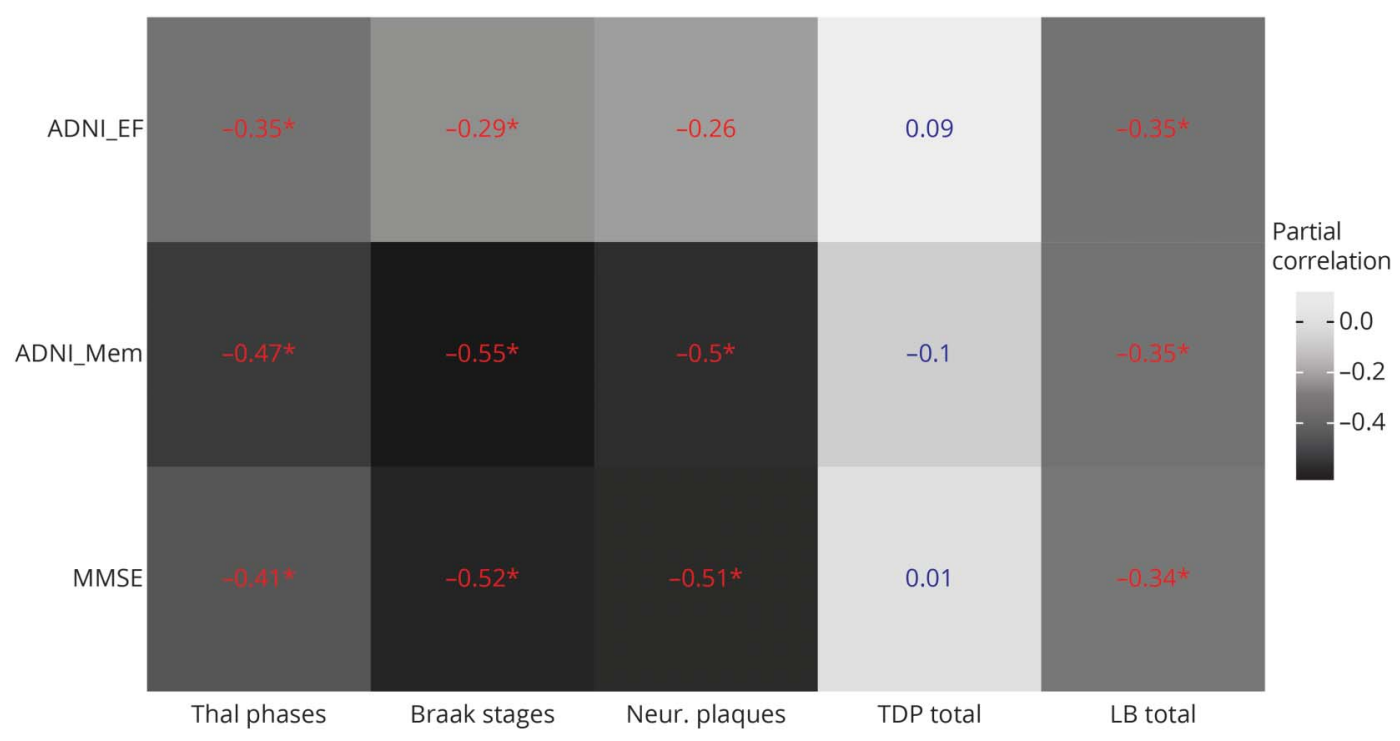

Partial correlations between cognitive measures and global neuropathologic scores, controlling for age, sex, and the interval between cognitive testing and death Numbers in blue indicate nonsignificant effects, numbers in red indicate significant effects at a $p$ value $<0.05$, uncorrected for multiple comparisons, and numbers in red with an * indicate significant effects at a false discovery rate-corrected $p$ value $<0.05$. ADNI_EF = ADNI composite executive function score; $A D N I \_M e m=A D N I$ composite memory score; Braak stages = Braak stages for neurofibrillary pathology; LB total = presence of Lewy body pathology (assessed according to dementia with Lewy bodies criteria as brainstem predominant, limbic, neocortical, amygdala predominant), yes/no; MMSE = Mini-Mental State Examination; Neur. plaques = Consortium to Establish a Registry for Alzheimer's Disease score for density of neocortical neuritic plaques; TDP total = presence of TDP-43 pathology (assessed in the following regions: spinal cord, amygdala, hippocampus, entorhinal cortex, neocortical), yes/no; Thal phases = Thal phases for amyloid plaques.

predominant TDP-43 pathology in older individuals that has recently been termed limbic-predominant age-related TDP43 encephalopathy (LATE). ${ }^{46}$

Braak stages had no significant effects in the ROI- or voxelbased analyses. A previous voxel-based analysis found a significant difference in the degree of atrophy between Braak V/ VI stages and Braak 0-II stages, but not between Braak III/IV stages and Braak 0-II stages. ${ }^{25}$ Different from this previous study, we could not find a significant difference in degree of atrophy between Braak V/VI vs Braak 0-II stages. We cannot explain this discrepancy. The number of participants within the different stages was comparable between the previous $(\mathrm{n}=$ 20 Braak 0-II and $n=59$ Braak V-VI) and our study ( $n=16$ Braak 0-II and $n=43$ Braak V-VI). Different from the previous study, we also controlled for the temporal interval between MRI and death. However, even when we removed this covariate we still did not find robust effects.

Our study has strengths and limitations. One major limitation is the lack of assessment of CAl neuron loss in the ADNI autopsy data that hindered a comprehensive evaluation of the role of CA1 degeneration, which was found to be a key driver of hippocampal atrophy in previous studies. ${ }^{35}$ However, according to neuropathologic Braak staging, neurofibrillary degeneration of CAl and the entorhinal cortex should be highly collinear. Another limitation is that the pathologic assessment of the $\mathrm{NbM}$ was only sampled from a single coronal section at the level of the crossing of the anterior commissure and did not selectively assess cholinergic neurons, preventing any inference on specific subsections of the NbM. A further limitation was the quality of the MRI scans. Due to the advanced stage of disease in this autopsy cohort, some of the cases exhibited severe brain atrophy, which can affect the quality of brain segmentation. This led to the exclusion of 2 of the 64 cases from the analysis due to insufficient scan quality for brain segmentation. Finally, the overrepresentation of men in the current cohort limits its translational value to aged cohorts where women are known to predominate.

A strength of our study is the regional assessment of neuropathology-brain volume associations, including standardized global neuropathologic rating scales and detailed local assessments of neuropathology within our target regions as well as ROI-based and complementary voxel-wise analyses of GM volume. Particularly, specific regional assessments of NbM pathology had not been examined yet in neuropathology-imaging correlation studies. Voxel-based analysis has only been used in a few previous studies on associations between MRI volumes and quantitative neuropathologic features ${ }^{25,39,47,48}$ or pathologically confirmed diagnoses. ${ }^{49,50}$ Here, we complemented our primary analyses focused on BF and MTL ROIs with a comprehensive voxel-wise search for associations of $\mathrm{AD}$ and non- $\mathrm{AD}$ neuropathologic features with brain-wide GM volume reductions.

The current findings confirm previous evidence for an association of $\mathrm{BF}$ volume with deposition of amyloid in cortical association areas, ${ }^{13,14}$ representing input areas of cholinergic 
projections. At the same time, LB pathology, but not NFT pathology, was associated with $\mathrm{BF}$ atrophy, pointing to the high relevance of pathologic comorbidity for the expression of regional atrophy in the $\mathrm{AD}$ spectrum. The validity of these findings is supported by the robust replication of previous reports on the association of TDP-43 pathology with hippocampal atrophy in $\mathrm{AD}$ cases. ${ }^{43}$ The question of traceability of a biomarker linking the in vivo measured marker with the neuropathologic reference standard is of high relevance for the $\mathrm{AD}$ field to enhance our ability to identify treatment targets and measure target engagement with valid biomarkers.

\section{Acknowledgment}

M.J.G. is supported by the "Miguel Servet" program [CP19/ 00031] of the Spanish Instituto de Salud Carlos III (ISCIIIFEDER). The authors thank Manuela Neumann, Department of Neuropathology of the University Medicine Tübingen, Germany, for discussions.

\section{Study funding}

Data collection and sharing for this project was funded by the Alzheimer's Disease Neuroimaging Initiative (ADNI) (NIH grant U01 AG024904) and DOD ADNI (Department of Defense award number W81XWH-12-2-0012). ADNI is funded by the National Institute on Aging, the National Institute of Biomedical Imaging and Bioengineering, and through contributions from the following: Alzheimer's Association; Alzheimer's Drug Discovery Foundation; Araclon Biotech; BioClinica, Inc.; Biogen Idec Inc.; Bristol-Myers Squibb Company; Eisai Inc.; Elan Pharmaceuticals, Inc.; Eli Lilly and Company; EuroImmun; F. Hoffmann-La Roche Ltd. and its affiliated company Genentech, Inc.; Fujirebio; GE Healthcare; IXICO Ltd.; Janssen Alzheimer Immunotherapy Research \& Development, LLC; Johnson \& Johnson Pharmaceutical Research \& Development LLC; Medpace, Inc.; Merck \& Co., Inc.; Meso Scale Diagnostics, LLC; NeuroRx Research; Neurotrack Technologies; Novartis Pharmaceuticals Corporation; Pfizer Inc.; Piramal Imaging; Servier; Synarc Inc.; and Takeda Pharmaceutical Company. The Canadian Institutes of Health Research is providing funds to support ADNI clinical sites in Canada. Private sector contributions are facilitated by the Foundation for the National Institutes of Health (fnih.org). The grantee organization is the Northern California Institute for Research and Education, and the study is coordinated by the Alzheimer's Disease Cooperative Study at the University of California, San Diego. ADNI data are disseminated by the Laboratory for Neuro Imaging at the University of Southern California.

\section{Disclosure}

The authors report no disclosures relevant to the manuscript. Go to Neurology.org/N for full disclosures.

\section{Publication history}

Received by Neurology November 26, 2019. Accepted in final form March 9, 2020.
Appendix 1 Authors

\begin{tabular}{lll}
\hline Name & Location & Contribution \\
\hline $\begin{array}{l}\text { Stefan J. } \\
\text { Teipel, MD }\end{array}$ & $\begin{array}{l}\text { University Medicine } \\
\text { Rostock and DZNE, } \\
\text { Germany }\end{array}$ & $\begin{array}{l}\text { Designed and conceptualized } \\
\text { study, analyzed the data, } \\
\text { drafted the manuscript for } \\
\text { intellectual content }\end{array}$ \\
\hline $\begin{array}{l}\text { H.- } \\
\text { Christian } \\
\text { Fritz, BSc }\end{array}$ & $\begin{array}{l}\text { University Medicine } \\
\text { Rostock and DZNE, }\end{array}$ & $\begin{array}{l}\text { Interpreted the data, revised } \\
\text { the manuscript for intellectual } \\
\text { content }\end{array}$ \\
\hline $\begin{array}{l}\text { Michel J. } \\
\text { Grothe, } \\
\text { PhD }\end{array}$ & DZNE, Germany & $\begin{array}{l}\text { Designed and conceptualized } \\
\text { study, helped in analyzing the } \\
\text { data, interpreted the data, } \\
\text { revised the manuscript for } \\
\text { intellectual content }\end{array}$ \\
\hline
\end{tabular}

Appendix 2 Coinvestigators

Coinvestigators are listed at links.Iww.com/WNL/B160

\section{References}

1. Dubois B, Feldman HH, Jacova C, et al. Advancing research diagnostic criteria for Alzheimer's disease: the IWG-2 criteria. Lancet Neurol 2014;13:614-629.

2. Dallaire-Theroux C, Callahan BL, Potvin O, Saikali S, Duchesne S. Radiologicalpathological correlation in Alzheimer's disease: systematic review of antemortem magnetic resonance imaging findings. J Alzheimers Dis 2017;57:575-601.

3. Bohnen NI, Grothe MJ, Ray NJ, Muller M, Teipel SJ. Recent advances in cholinergic imaging and cognitive decline: revisiting the cholinergic hypothesis of dementia. Curr Geriatr Rep 2018;7:1-11.

4. Kilimann I, Grothe M, Heinsen $\mathrm{H}$, et al. Subregional basal forebrain atrophy in Alzheimer's disease: a multicenter study. J Alzheimers Dis 2014;40:687-700.

5. Grothe M, Heinsen H, Teipel SJ. Atrophy of the cholinergic basal forebrain over the adult age range and in early stages of Alzheimer's disease. Biol Psychiatry 2012;71: 805-813.

6. Mesulam MM. The systems-level organization of cholinergic innervation in the human cerebral cortex and its alterations in Alzheimer's disease. Prog Brain Res 1996, 109:285-297.

7. Schmitz TW, Nathan Spreng R; Alzheimer's Disease Neuroimaging Initiative. Basal forebrain degeneration precedes and predicts the cortical spread of Alzheimer's pathology. Nat Commun 2016;7:13249.

8. Mufson EJ, Ginsberg SD, Ikonomovic MD, DeKosky ST. Human cholinergic basal forebrain: chemoanatomy and neurologic dysfunction. J Chem Neuroanat 2003;26: 233-242.

9. Mesulam M. Cholinergic aspects of aging and Alzheimer's disease. Biol Psychiatry 2012;71:760-761.

10. Liu AK, Chang RC, Pearce RK, Gentleman SM. Nucleus basalis of Meynert revisited: anatomy, history and differential involvement in Alzheimer's and Parkinson's disease. Acta Neuropathol 2015;129:527-540.

11. Cykowski MD, Takei H, Van Eldik LJ, et al. Hippocampal sclerosis but not normal aging or Alzheimer disease is associated with TDP-43 pathology in the basal forebrain of aged persons. J Neuropathol Exp Neurol 2016;75:397-407.

12. Potter PE, Rauschkolb PK, Pandya Y, et al. Pre- and post-synaptic cortical cholinergic deficits are proportional to amyloid plaque presence and density at preclinical stages of Alzheimer's disease. Acta Neuropathol 2011;122:49-60.

13. Grothe MJ, Ewers M, Krause B, Heinsen H, Teipel SJ; Alzheimer's Disease Neuroimaging Initiative. Basal forebrain atrophy and cortical amyloid deposition in nondemented elderly subjects. Alzheimers Dement 2014;10:S344-S353.

14. Kerbler GM, Fripp J, Rowe CC, et al. Basal forebrain atrophy correlates with amyloid beta burden in Alzheimer's disease. Neuroimage Clin 2015;7:105-113.

15. McKhann G, Drachman D, Folstein M, Katzman R, Price D, Stadlan EM. Clinical diagnosis of Alzheimer's disease: report of the NINCDS-ADRDA Work Group under the auspices of the Department of Health and Human Services Task Force on Alzheimer's disease. Neurology 1984;34:939-944.

16. Franklin EE, Perrin RJ, Vincent B, et al. Brain collection, standardized neuropathologic assessment, and comorbidity in Alzheimer's Disease Neuroimaging Initiative 2 participants. Alzheimers Dement 2015;11:815-822.

17. Montine TJ, Phelps CH, Beach TG, et al. National Institute on Aging-Alzheimer's Association guidelines for the neuropathologic assessment of Alzheimer's disease: a practical approach. Acta Neuropathol 2012;123:1-11.

18. Folstein MF, Folstein SE, McHugh PR. Mini-mental-state: a practical method for grading the cognitive state of patients for the clinician. J Psychiatr Res 1975;12: 189-198. 
19. Crane PK, Carle A, Gibbons LE, et al. Development and assessment of a composite score for memory in the Alzheimer's Disease Neuroimaging Initiative (ADNI). Brain Imaging Behav 2012;6:502-516.

20. Gibbons LE, Carle AC, Mackin RS, et al. A composite score for executive functioning validated in Alzheimer's Disease Neuroimaging Initiative (ADNI) participants with baseline mild cognitive impairment. Brain Imaging Behav 2012;6:517-527.

21. Ashburner J. A fast diffeomorphic image registration algorithm. NeuroImage 2007;38: 95-113.

22. Wolf D, Bocchetta M, Preboske GM, Boccardi M, Grothe MJ; Alzheimer's Disease Neuroimaging Initiative. Reference standard space hippocampus labels according to the EADC-ADNI harmonized protocol: utility in automated volumetry. Alzheimers Dement 2017;13:893-902.

23. Hammers A, Allom R, Koepp MJ, et al. Three-dimensional maximum probability atlas of the human brain, with particular reference to the temporal lobe. Hum Brain Mapp 2003;19:224-247.

24. Benjamini Y, Hochberg Y. Controlling the false discovery rate: a practical and powerful approach to multiple testing. J Roy Stat Soc B Met 1995;57:289-300.

25. Whitwell JL, Josephs KA, Murray ME, et al. MRI correlates of neurofibrillary tangle pathology at autopsy: a voxel-based morphometry study. Neurology 2008;71:743-749.

26. Baker-Nigh A, Vahedi S, Davis EG, et al. Neuronal amyloid-beta accumulation within cholinergic basal forebrain in ageing and Alzheimer's disease. Brain 2015;138:1722-1737.

27. Kolisnyk B, Al-Onaizi M, Soreq L, et al. Cholinergic surveillance over hippocampa RNA metabolism and Alzheimer's-like pathology. Cereb Cortex 2017;27:3553-3567.

28. Brenowitz WD, Keene CD, Hawes SE, et al. Alzheimer's disease neuropathologic change, Lewy body disease, and vascular brain injury in clinic- and community-based samples. Neurobiol Aging 2017;53:83-92.

29. Mesulam M, Shaw P, Mash D, Weintraub S. Cholinergic nucleus basalis tauopathy emerges early in the aging-MCI-AD continuum. Ann Neurol 2004;55:815-828.

30. Geula C, Nagykery N, Nicholas A, Wu CK. Cholinergic neuronal and axonal abnormalities are present early in aging and in Alzheimer disease. J Neuropathol Exp Neurol 2008;67:309-318.

31. Mufson EJ, Ikonomovic MD, Counts SE, et al. Molecular and cellular pathophysiology of preclinical Alzheimer's disease. Behav Brain Res 2016;311:54-69.

32. Silbert LC, Quinn JF, Moore MM, et al. Changes in premorbid brain volume predict Alzheimer's disease pathology. Neurology 2003;61:487-492.

33. Csernansky JG, Hamstra J, Wang L, et al. Correlations between antemortem hippocampal volume and postmortem neuropathology in $\mathrm{AD}$ subjects. Alzheimer Dis Assoc Disord 2004;18:190-195.

34. Serrano-Pozo A, Qian J, Muzikansky A, et al. Thal amyloid stages do not significantly impact the correlation between neuropathological change and cognition in the Alzheimer disease continuum. J Neuropathol Exp Neurol 2016;75:516-526.

35. Zarow C, Vinters HV, Ellis WG, et al. Correlates of hippocampal neuron number in Alzheimer's disease and ischemic vascular dementia. Ann Neurol 2005;57:896-903.
36. Apostolova LG, Zarow C, Biado K, et al. Relationship between hippocampal atrophy and neuropathology markers: a 7T MRI validation study of the EADC-ADNI Harmonized Hippocampal Segmentation Protocol. Alzheimers Dement 2015;11: 139-150.

37. Bobinski M, de Leon MJ, Wegiel J, et al. The histological validation of post mortem magnetic resonance imaging-determined hippocampal volume in Alzheimer's disease. Neuroscience 2000;95:721-725.

38. Thaker AA, Weinberg BD, Dillon WP, et al. Entorhinal cortex: antemortem cortical thickness and postmortem neurofibrillary tangles and amyloid pathology. AJNR Am J Neuroradiol 2017;38:961-965.

39. Josephs KA, Whitwell JL, Weigand SD, et al. TDP-43 is a key player in the clinical features associated with Alzheimer's disease. Acta Neuropathol 2014;127:811-824.

40. Robinson AC, Davidson YS, Horan MA, Pendleton N, Mann DMA. Pathological correlates of cognitive impairment in the University of Manchester Longitudinal Study of cognition in normal healthy old age. J Alzheimers Dis 2018;64:483-496.

41. Josephs KA, Whitwell JL, Tosakulwong N, et al. TAR DNA-binding protein 43 and pathological subtype of Alzheimer's disease impact clinical features. Ann Neurol 2015; 78:697-709.

42. James BD, Wilson RS, Boyle PA, Trojanowski JQ, Bennett DA, Schneider JA. TDP-43 stage, mixed pathologies, and clinical Alzheimer's-type dementia. Brain 2016;139: 2983-2993.

43. Josephs KA, Dickson DW, Tosakulwong N, et al. Rates of hippocampal atrophy and presence of post-mortem TDP-43 in patients with Alzheimer's disease: a longitudinal retrospective study. Lancet Neurol 2017;16:917-924.

44. Ihara R, Vincent BD, Baxter MR, et al. Relative neuron loss in hippocampal sclerosis of aging and Alzheimer's disease. Ann Neurol 2018;84:741-753.

45. Makkinejad N, Schneider JA, Yu J, et al. Associations of amygdala volume and shape with transactive response DNA-binding protein 43 (TDP-43) pathology in a community cohort of older adults. Neurobiol Aging 2019;77:104-111.

46. Nelson PT, Dickson DW, Trojanowski JQ et al. Limbic-predominant age-related TDP-43 encephalopathy (LATE): consensus working group report. Brain 2019;142: $1503-1527$.

47. Kantarci K, Murray ME, Schwarz CG, et al. White-matter integrity on DTI and the pathologic staging of Alzheimer's disease. Neurobiol Aging 2017;56:172-179.

48. Josephs KA, Whitwell JL, Knopman DS, et al. Abnormal TDP-43 immunoreactivity in $\mathrm{AD}$ modifies clinicopathologic and radiologic phenotype. Neurology 2008;70: $1850-1857$.

49. Hornberger $\mathrm{M}$, Wong $\mathrm{S}$, Tan $\mathrm{R}$, et al. In vivo and post-mortem memory circuit integrity in frontotemporal dementia and Alzheimer's disease. Brain 2012;135: 3015-3025.

50. Harper L, Bouwman F, Burton EJ, et al. Patterns of atrophy in pathologically confirmed dementias: a voxelwise analysis. J Neurol Neurosurg Psychiatry 2017;88 908-916. 


\section{Neurology}

Neuropathologic features associated with basal forebrain atrophy in Alzheimer disease Stefan J. Teipel, H.-Christian Fritz, Michel J. Grothe, et al. Neurology 2020;95; e1301-e1311 Published Online before print July 6, 2020

DOI 10.1212/WNL.0000000000010192

This information is current as of July 6, 2020

\section{Updated Information \& Services}

References

Citations

Subspecialty Collections

Permissions \& Licensing

Reprints including high resolution figures, can be found at: http://n.neurology.org/content/95/10/e1301.full

This article cites 50 articles, 6 of which you can access for free at: http://n.neurology.org/content/95/10/e1301.full\#ref-list-1

This article has been cited by 2 HighWire-hosted articles: http://n.neurology.org/content/95/10/e1301.full\#\#otherarticles

This article, along with others on similar topics, appears in the following collection(s):

\section{Alzheimer's disease}

http://n.neurology.org/cgi/collection/alzheimers_disease

\section{Memory}

http://n.neurology.org/cgi/collection/memory

MRI

http://n.neurology.org/cgi/collection/mri

Information about reproducing this article in parts (figures,tables) or in its entirety can be found online at:

http://www.neurology.org/about/about_the_journal\#permissions

Information about ordering reprints can be found online:

http://n.neurology.org/subscribers/advertise

Neurology ${ }^{\circledR}$ is the official journal of the American Academy of Neurology. Published continuously since 1951, it is now a weekly with 48 issues per year. Copyright Copyright ( 2020 The Author(s). Published by Wolters Kluwer Health, Inc. on behalf of the American Academy of Neurology.. All rights reserved. Print ISSN: 0028-3878. Online ISSN: 1526-632X.

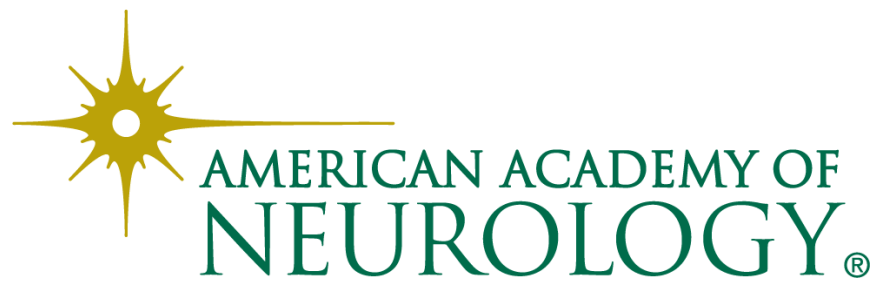

